\title{
Thermal Energy Storage Selection for Near Term Nuclear Integration
}

\author{
Daniel Mark Mikkelson, Konor L Frick, \\ Shannon M Bragg-Sitton, Joseph Michael \\ Doster, Elizabeth Kirkpatrick Worsham
}

November 2019

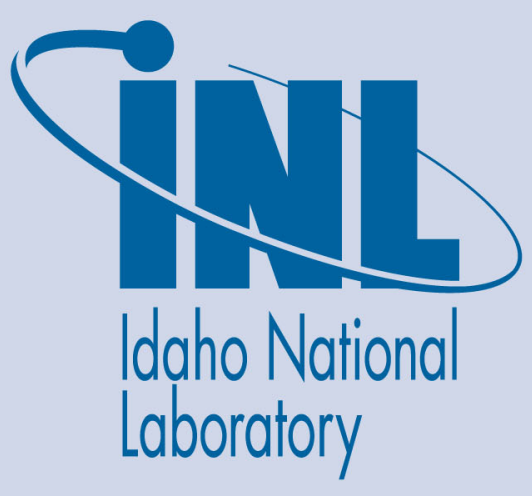

The INL is a U.S. Department of Energy National Laboratory operated by Battelle Energy Alliance 


\section{Thermal Energy Storage Selection for Near Term Nuclear Integration}

Daniel Mark Mikkelson, Konor L Frick, Shannon M Bragg-Sitton, Joseph Michael Doster, Elizabeth Kirkpatrick Worsham

November 2019

Idaho National Laboratory Idaho Falls, Idaho 83415

http://www.inl.gov

Prepared for the

U.S. Department of Energy

Under DOE Idaho Operations Office

Contract DE-AC07-05ID14517 


\title{
Thermal Energy Storage Selection for Near Term Nuclear Integration
}

\author{
Daniel Mikkelson*, Konor Frick* ${ }^{*}$ J. Michael Doster*, Shannon Bragg-Sitton ${ }^{*}$, Elizabeth Worsham* \\ *North Carolina State University Department of Nuclear Engineering, 2500 Stinson Dr, Raleigh, 27695, \\ dmmikkel@ncsu.edu,doster@ncsu.edu,ekworsha@ncsu.edu \\ 'Idaho National Laboratory, 2525 Fremont Ave, Idaho Falls, 84302, konor.frick@inl.gov,.Shannon.bragg-sitton@inl.gov
}

\section{INTRODUCTION}

Thermal energy storage (TES) integration with existing and future light water reactors (LWRs) has the capability to increase the deployment options and uses for the large amounts of heat produced in nuclear reactor cores. The present method of exclusively producing electricity has become less economical with increases in renewable energy penetration leading to times of low or negative electricity prices. Some response to this new grid reality has been to begin load following nuclear reactors, an unideal choice due to the relatively small modular cost of producing nuclear energy compared to the revenue that could be received by using all of the heat produced.

To support research in this area, Idaho National Laboratory (INL) has joined with Utah Associated Municipal Power Systems (UAMPS) on the Carbon Free Power Project (CFPP) to contract the Joint Use Modular Plant (JUMP). CFPP is planned to be a 12-module NuScale operating site built on INL property, with the first module dedicated to JUMP for research, development, and demonstration purposes. The JUMP module coming online around the beginning of fiscal year 2027 in the current project timeline. One primary goal of JUMP is to demonstrate TES integrated with an operating LWR in a real-world environment. TES integration will allow for the demonstration of nuclear power's capability to participate in grid load following without reactor cycling, process heat integration, and electrical ancillary market opportunities. The goal of this research is to support both SMR deployment opportunities as well as support LWR retrofit capabilities.

In a discussion limited to load following or energy arbitration, "charging" and "discharging" can be simply defined. TES charging refers to time when energy is removed from the nuclear reactor power conversion cycle and directed to be stored in the TES storage medium resulting in less available energy available for grid output. Discharging refers to the process of releasing energy stored in the TES medium and having an increase in grid.

\section{Thermal Energy Storage Options}

Ten different technologies, with thirteen total implementations, have been evaluated. Underground thermal energy storage, hot \& cold water storage, geothermal energy storage, concrete energy storage, firebrick energy storage, phase change materials, thermochemical energy storage, thermocline liquid sensible heat storage, 2-tank liquid sensible heat storage, and steam accumulators are considered. A brief description of each technology is contained in this section.

Underground thermal energy storage (UTES) uses aquifer structures or borehole insertion. Current experience with UTES is focused in seasonal storage for domestic heating and cooling, and for HVAC applications. The storage uses hot and cold water that is exchanged between hot and cold stores, using the environment as the primary heat storage medium.

Hot \& cold water storage (HCWS) is characterized as large tanks of hot or chilled water stored above ground and cycled typically on a daily basis. Chillers can use either waste heat or low-demand time electricity to produce a local reservoir of cold water. Hot water is produced via heat exchangers and stored below saturation temperature in order to avoid pressure concerns. Note that in this work, HCWS is considered as a separate technology from steam accumulators.

Geothermal energy storage (GeoTES) is similar to UTES, except that the storage is much deeper in the ground and the primary storage medium is steam and water. By creating an aquifer-like structure at depths where the hydrostatic pressure will not cause fracturing, it is possible to store water or steam deep in the earth at relatively high temperatures and pressures. Example depths of at least $2 \mathrm{~km}$ allows for saturation temperatures around $250^{\circ} \square$.

Concrete energy storage uses specialized concrete overlaid onto tubing lattices to transfer heat from a heat transfer fluid (HTF) into the surrounding concrete. A cold HTF is used to recover heat from the concrete. Concrete is normally used as an insulator, and so material and design considerations must account for a slower rate of heat flow through the storage medium itself during charging and discharging.

Firebrick thermal energy storage (FIRES) uses electrical resistance heated ceramic bricks to store very low or negatively priced electricity to resell at higher priced times via air heated by the firebrick. If possible, the firebrick system will optimally operate at a $1000 \square$ temperature from 850-1850 $\square$. Firebrick discharge will either be used for direct thermal energy or to produce electricity via a Brayton cycle.

Phase change materials (PCMs) take advantage of latent heat processes to store heat primarily via a change in material phase. The primary technology investigated in 
research is solid-liquid material change. PCMs face considerable material considerations and limitations. Research continues because of very high energy densities and constant temperature processes in PCMs.

Thermochemical energy storage (TCS) uses heat to initiate reversible endothermic chemical reactions. The products of the charging process are then the reactants of the discharge process. TCS techniques require the ability to transfer heat into the reactants, separate the products for storage, and then recombine the stored products to become reactants. Similar to PCMs, there are a great deal of material considerations involved in these systems.

Thermocline liquid sensible heat storage (TCSHS) takes advantage of buoyancy and low internal heat transfer characteristics to store hot and cold liquid in a single tank separated by a thin but steep thermal gradient layer. This thermocline would move up and down in the tank during discharging and charging. Water is currently used in thermocline systems, and research is being done for other materials. Specific mixing, material considerations, and thermocline reduction are all areas of concern in TCSHS.

Two-Tank liquid sensible heat storage (TTSHS) uses two large stores of the storage medium to store heat. During charging, the storage liquid is pumped from the cold tank through a heat exchanger to be stored in the hot tank. While discharging, the reverse is done. Storage pressure is maintained via gases at the top of the two tanks. Most current TES experience in two-tank systems is in the concentrated solar industry using molten salts as a storage medium.

Steam accumulators (SAs) store saturated water in a sliding pressure Ruths type vessel. During charging, steam is injected in the bottom of the tank system via diffusers. The system mixes into a saturated system, pressurized by the steam volume in the upper section. During discharge, a valve is opened allowing for steam to escape. The ensuing vacuum in the top of the vessel lowers the pressure, causing some liquid to flash to steam. Thus, during discharge the pressure out of SAs decreases.

\section{Thermal Energy Storage Applications}

The most direct TES application is grid load following or energy arbitration. In this mode, the TES would charge during times of either low demand or low energy price. The TES would then discharge during times of high demand or high energy price. Regardless of the motivation, system energy output is depressed in one time period in order to be peaked later. Many studies have focused on these two applications as the primary goal of energy storage. To obtain TES charging fluid with the highest energy content, current proposals for TES integration with LWRs use a turbine bypass valve, moving steam at its highest energy point after the reactor steam generators. After storage, steam reintroduction into the nuclear power conversion process would depend on turbine design and available temperature and pressure coming out of the TES. It is commonly discussed to reintroduce this peaking steam before the low pressure turbine.

Alternatively, TES should allow nuclear reactors to directly provide heat to various processes. TES would introduce a physical separation between nuclear safety components and an outside safety risk. Processes that nuclear energy could couple to depend then on three main factors: the temperature and pressure of nuclear steam output, the pressures and temperatures needs of the outside process, and the ability for TES to accept nuclear energy and discharge the process energy efficiently. Some example processes include high temperature steam electrolysis (HTSE), water desalination, pulp and paper processing. These three technologies represent a wide range of demands, and thus can be used as boundary conditions for research demonstration.

High temperature steam electrolysis uses high temperature steam (850 $\square$ ) and electricity to split water into hydrogen and oxygen. The goal of HTSE is hydrogen production for later use in fuel cell systems. To couple this system to nuclear steam processes, some amount of topping heat must be added into the system to obtain such high temperatures. It is possible to do this with electrical heaters power by the nuclear reactor.

Water desalination is primarily done via reverse osmosis (RO). Most of the demand of this system is electrical. The main thermal component is feedstock preheating. Both processes could be done using a nuclear reactor. Due to the increasing global water crisis, nuclear integration with desalination has gained traction.

Pulp and paper processing is investigated in this research as an example chemical process integrable with LWR technology. Pulp and paper mills use on-site turbines operating at $2.7 \mathrm{MPa}$ (400 psia), which nuclear heat could feed directly into. Pulp and paper mills require a significant amount of off-site steam production for various processes. They can also be used as an example case for any chemical process requiring steam at $2.7 \mathrm{MPa}$ or less.

\section{Figures of Merit}

Thirteen categories were identified as important criteria for TES selection. These categories were created prior to the evaluation of the technologies to help objectivity. Eleven of these categories have been assigned with numerical evaluations of their value to JUMP and LWR integration. The figures of merit (FOM) evaluation gives numerical values based on criteria discussed below.

1) Technology readiness level (TRL) is extremely important to JUMP as the system must be fully designed in the next few years to be licensed and deployed by 2027 . The FOM value equals the TRL.

2) Experienced technology integration with nuclear steam pressures and temperatures. The NuScale reactor maintains steam pressure of $2.7 \mathrm{MPa}$ and $300 \square$, Being able 
to directly use this steam achieves a FOM of 2, having to somewhat downgrade this steam gives a FOM of 1, and a FOM of 0 indicates no direct use of this steam.

3) Capability to discharge high quality heat. The TES must be able to discharge its energy capacity at consistent high quality heat. A system capable of discharging its entire store at above $175 \square$ was given a FOM of 2. A system capable of either sliding pressure discharge with some above $175 \square$ or a system capable of discharging between 100-175 $\square$ was given a FOM of 1. Otherwise, the FOM was 0 for this category.

4) United States Electrical grid ancillary services market potential. If a TES allows for participation in the frequency regulation market, a FOM of 2 was given. If a TES allows for participation in the reserves market, a FOM of 1 was given. Otherwise, a 0 was given for this category.

5) Total energy capacity for this system is estimated at $400 \mathrm{MWh}$ of heat. A system must therefore be able to manage that much energy. A system capable of this size was given a FOM of 2. One that could store 100MWh but not $400 \mathrm{MWh}$ was given a 1 , and a 0 was given otherwise.

6) Ramp time is associated with category 4 , as well as energy arbitration and load following. Due to the most restrictive reserve market restrictions of 10 minutes, a ramp time to maximum power of 10 minutes or less was given a FOM of 2. A ramp time of less than an hour is assigned a 1, and any ramp longer than that is given a 0 .

7) Cycle frequency, defined as the system's capability to charge and discharge. If a system can charge and discharge at will, a FOM of 2 was given. A system that could cycle only daily is given a FOM of 1 , and a 0 to systems with longer cycles.

8) Realignment frequency is the need of a system to either wait on some phenomenon or correct a non-ideal process and leads to a reduction in capacity factor. If no realignment is required, a FOM of 2 is given. If realignment is required every cycle, a FOM of 0 is given, and a FOM of 1 is given for values in the middle.

9) TES cost per $\mathrm{kWh}$. This value is evaluated after the total FOM is calculated, to be used as a selection method later.

10) Technology lifetime refers to the time before the TES would need self-replacement. Like cost, this factor is considered after the FOM is calculated.

11) Geographical insensitivity. Because it is desired for SMR with TES to be deployed globally with as little redesign as possible, geographical needs are considered. A FOM of 0 is given to a geographic specific technology, and a FOM of 1 is given to a TES without those requirements.

12) Environmental concerns are important and can appear in multiple stages of TES use: construction, direct use, and during deconstruction. A FOM of 1 is given to a technology that does not have any significant environmental concerns, and a FOM of 0 was given to a technology with environmental concerns.
13) Minimum turndown or thermal support requirements. A system that needs heat tracing or a constant supply of heat is given a FOM of 0 . A system that faces no concerns if heat is not constantly supplied is given a FOM of 1 .

\section{RESULTS}

The results show satisfactory stratification of technology options using the FOM system developed. A maximum FOM value of 26 was not achieved by any technology, but concrete, TTSHS, and SAs each approached the value with FOM totals of 20 and higher. The average FOM total was 16.8. The FOM achieved its goal of identifying the most appropriate technologies for the project. Additionally, the specific criteria within FOM categories can be easily altered to allow for future use with various specific applications.

Table 1. TES FOM evaluations.

\begin{tabular}{|c|c|c|c|}
\hline Technology & FOM & Technology & FOM \\
\hline UTES & 14 & HCWS & 18 \\
\hline GeoTES & 11 & Concrete & 20 \\
\hline FIRES & 14 & PCMs & 16 \\
\hline TCS & 12 & TCSHS & 17 \\
\hline TTSHS & 23 & SAs & 22 \\
\hline Maximum FOM: & 26 & Average FOM: & 16.7 \\
\hline
\end{tabular}

The FOM results clearly indicate that either concrete, TTSHS, or SAs should be identified as the preferred storage technology for the JUMP project. With this initial reduction in options, a second and more qualitative selection can be done focusing on specific advantages and disadvantages of the technologies.

Concrete has a significant advantage in system cost. The casing of piping in specialized cast concrete structures should be cheap, as both materials are readily available. However, laboratory experiments and simulations of solid media systems, including concrete, have shown that the storage efficiency of these systems is low. Namely, that only around $70 \%$ of heat that is charged is available for discharge. Additionally, there is not a separation between power and energy. Like batteries, the maximum power output is limited by the current energy content.

Steam accumulators have very impressive response times to changes in output demand. The positive impact of this reaction speed is unfortunately balanced by the sliding pressure of steam accumulators. Some constant pressure accumulator designs exist, but were not evaluated due to their low TRL relative to existing SAs. The other limiting factor for SAs is their price. Already considered expensive individually, a $400 \mathrm{MWh}$ system based on steam accumulators would likely require around $23,000 \mathrm{~m}^{3}$ of capacity, needing around 600 individual accumulators connected in parallel. 
Two-tank sensible heat storage has the highest FOM value due to its current use in molten salt systems, disconnect between power capacity and energy stored, and relative cost efficiency impacted by its storage efficiency. Conceptually simple, TTSHS is a viable short term selection option for JUMP. Material selection for a two-tank sensible heat system is the most logical next step. Using solar power as an example, thermal oils and molten salts are the most widely used HTFs and storage media. Due to these two options and their separate cost considerations, they will be looked at separately, and "molten salt" and "thermal oil" now refer to two-tank configurations only.

Table 2. FOM and Cost of down-selected TES options

\begin{tabular}{|c|c|c|}
\hline Technology & FOM & Est. Cost $(\$ / \mathrm{kWh})$ \\
\hline Concrete & 20 & $25-150$ \\
\hline Molten Salt & 23 & $5-20$ \\
\hline Thermal Oil & 23 & $8-16$ \\
\hline SAs & 22 & $130-400$ \\
\hline
\end{tabular}

Based on the FOM evaluation, subsequent qualitative assessment, and estimated cost, two-tank sensible heat storage using a low temperature molten salt is recommended for the TES component for the JUMP module. The system should be able to provide the most flexibility with the best cost of all options evaluated herein. Without additional analysis using LWR parameters, precise costs of molten salt storage and thermal oil storage cannot be immediately calculated.

The results of this down-selected TES system will provide input into the first of a kind nuclear TES system built in conjunction with the JUMP module. The methods discussed in this paper also provide guidance potential for future TES integrations with their own specific requirements. By increasing the deployment options of future SMRs, and diversifying the applications of existing LWRs.

\section{FUTURE WORK}

Detailed modeling of the selected TES is upcoming research at INL, with the intent to dynamically simulate the entire energy transport and conversion system of a NuScale reactor integrated with TES for various potential energy applications including, but not limited to, high temperature steam electrolysis and load following. Additionally, the initial cost matrix is indeterminate regarding which HTF should be used for nuclear integration, so a detailed cost analysis must be done for the two-tank systems at nuclear temperatures.

\section{ACKNOWLEDGEMENTS}

Funding for this research comes form the Light Water Reactor Sustainability, Integrated Energy Systems, and Joint Use Modular Plant research programs at Idaho National Laboratory.

\section{REFERENCES}

1. NuScale Standard Plant Design Certification Applicaiton, "Steam Power and Conversion System," (2008).

2. J. COLEMAN et al, "An evaluation of energy storage options for nuclear power," Nuclear Science and Technology, Idaho National Laboratory, INL/EST-1742420, 2017.

3. Z. ZHOU et al, "Survey of U.S. Ancillary Services Market," Argonne National Laboratory, ANL/ESD-16/1, 2016.

4. J. KIM et al, "Status report on the high-temperature steam electrolysis model developed in the Modelica framework (FYH2017)," Nuclear Science and Technology, Idaho National Laboratory, INL/EST-17-43056, 2017.

5. H. TRAN and E. VAKKILAINNEN, "The kraft chemical recovery process." Available: https://www.tappi.org/content/events/08kros/manuscripts/11.pdf

6. DEPARTMENT OF ENERGY, "Technology readiness assessment guide," DOE G 413.3-4A, 2001.

7. $\quad$ K. FRICK et al, "Modeling hybrid nuclear systems with chilled-water storage," Nuclear Technology 202, 5370, 2018.

8. SANDIA NATIONAL LABORATORY, "DOE Global Energy Storage Database"

9. WENDT et al, "Geologic thermal energy storage of solar heat to provide a source of dispatchable renewable power and seasonal energy storage capacity," GRC Transactions, 43, (2019).

10. STACK et al. "Performance of firebrick resistanceheated energy storage for industrial heat applications and round trip electricity storage," Applied Energy, 242, 782796, (2019).

11. N. HOIVIK et al, "Demonstration of EnergyNest thermal energy storage (TES) technology," Proc. IEEE, 100, (2012).

12. K. PIELICHOWSKA and K. PIELIECHOWSKI, "Phase change materials for thermal energy storage," Progress in Materials Science, 65, 67-123, (2014).

13. A. DE JONG et al, "Thermochemical storage system design issues," Energy Procedia, 48, 309-319 (2014).

14. G. ANGELINI et al, "Comparison of thermocline molten salt storage performances to commercial two-tank configuration," Energy Procedia, 49, 694-704, (2014).

15. M. DANIELS, "Integration of large-scale steam accumulators for energy storage in nuclear hybrid energy systems," North Carolina State University, Thesis, (2017). 16. OFFICE OF ELECTRICITY, "Department of energy global energy storage database," Sandia National Laboratory. 